\title{
Michaelis-Menten and beyond
}

\section{The 'textbook' enzyme kinetics plots, still part of the kinetics art.}

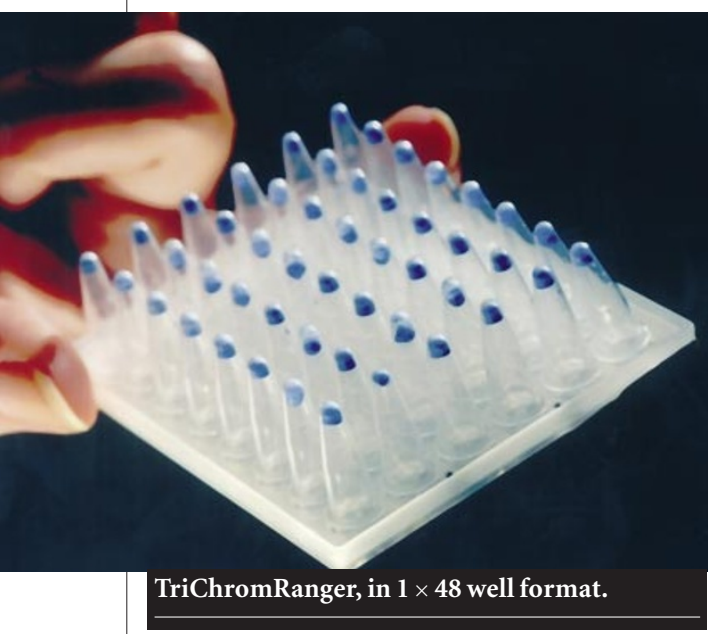

TriChromRanger

Pierce

www.piercenet.com

Ready-to-go molecular weight markers

TriChromRanger is a mixture of seven prestained proteins in the range $16.5-210 \mathrm{~K}$. The second highest molecular weight component (phosphorylase B) and the lowest (lysozyme) are covalently modified with red and purple dye, respectively, to act as reference bands within the gel. The five remaining proteins have been covalently modified with a blue dye. The inclusion of two differently stained bands in the marker allows easy marker protein recognition on Western blots or SDS-PAGE gels, should the dye front run off the bottom of the gel.

Enzyme kinetics module

SPSS www.spss.com

Custom graphing for SigmaPlot

This new module extends SigmaPlot's curvefitting and graphing capabilities. It features a data entry wizard for transferring data into a SigmaPlot worksheet, including material cutand-pasted from other software packages or entered manually. Replicate velocities may be entered and, for inhibition studies, substrate or inhibitor concentrations can be varied for each series. There is a built-in library of 11 equation groups and 35 model types. Graph types include Michaelis-Menten, LineweaverBurke, Eadie-Hofstee and Scatchard.

\section{QuantiPro}

Sigma-Aldrich www.sigma-aldrich.com High-sensitivity protein assay

QuantiPro is designed to assay protein concentrations down to $0.05 \mu \mathrm{gml}^{-1}$ with low sensitivity to interfering agents, a stable colour reaction and resistance to detergents. The bicinchoninic acid (BCA) assay relies on the protein-depen- dent reduction of copper under alkaline conditions. BCA forms a purple-blue complex with reduced copper in this alkaline environment. Applications include microtitre plate assay and determination of functional groups on solid supports and in solutions.

\section{CoreHTS}

PanVera

www.panvera.com Tyrosine kinase assay with a competitive edge

Fluorescence polarization is the detection method used in the 'Tyrosine Kinase Assay Kit, Green', which is designed for high-throughput drug discovery screening of novel tyrosine kinase inhibitors. The assay is based on competition between a fluorescein-labelled phosphopeptide tracer and the non-fluorescent phosphopeptides generated in the enzyme reaction, binding to an anti-phosphotyrosine antibody. The antibody and the fluorescent phosphopeptide tracer are supplied as separate components in the kit, which is available in 100, 1,000 and bulk assay sizes.

\section{Substrate profiling libraries}

\section{ChemRx}

www.chemrx.com New screening tools for protease and GPCR

The ChemRx tripeptidyl aminomethyl coumarin (AMC) library can be used in protease screening for novel substrates for known and new protease targets and to gain information on P1-P3 binding specificity for inhibitor development. And ChemRx's G-protein coupled receptor (GPCR) targeted library is designed for use as a screening tool in the identification oflead compounds.

\section{DuoLuX}

Vector Laboratories www.vectorlabs.com Alkaline phosphatase and peroxidase substrates Both these substrates can be used in 'blot assays' or in ELISA applications. Sensitivity in western blot chemiluminescent detection is approximately 1 pg of protein using either the phosphatase or peroxidase. For nucleic acid detection, the alkaline phosphatase substrate is slightly more sensitive than that for peroxidase, enabling visualization of 100 fg of target nucleic acid. Peroxidase substrate is supplied in two $100-\mathrm{ml}$ bottles, mixed to yield $200 \mathrm{ml}$ of substrate; alkaline phosphatase substrate is ready to use in a $100 \mathrm{ml}$ package.

\section{Freezer racks}

Nalgene www.nalgenunc.com Cryogenic storage for multiwell plates

Two new designs are available in Nalgene's range of freezer storage racks. A 16-shelf horizontalmicrowell platerack in $4 \times 4$ arrayholds 48 standard 96-well plates or 16 deep well plates, while a larger 24-shelf unit will accommodate 72 standard 96-well plates or 24 deep well plates. The stainless steel racks have a retainer clip to hold plates in place, top and side handles and a label holder.

These notes are compiled in the Nature office from information provided by the manufacturers.

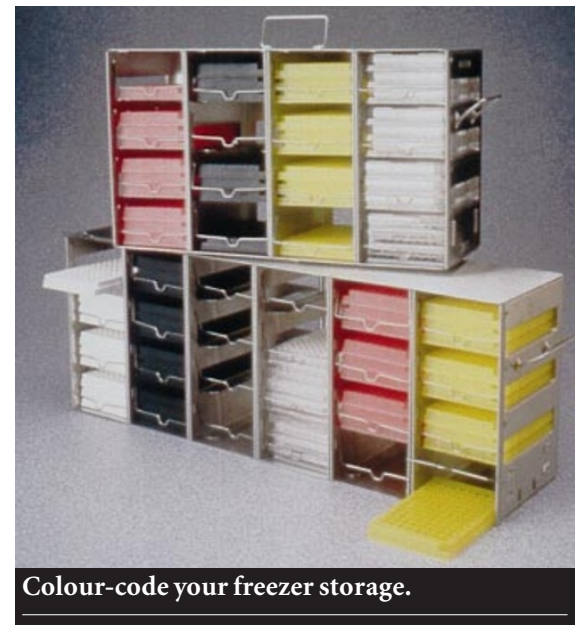

\section{ADVERTISEMENTS}

Polyclonal Antibodies $\&$ Custom Peptides

Please contact us about complete services for your custom peptide and polyclonal antibody needs.

\section{ResGen Invitrogen Corporation}

U.S. or Canada 800-533-4363 FAX 256-536-9016

Check out our web site at www.resgen.com
Ubiquitinylation assays?

Ub-activating enzyme E1 Ub-conjugating enzymes (E2s)

Fractions I \& || Ub conjugating kit

\section{ww.proteasomecom}

AfFinITI Research Products Ltd. Mamhead Castle, Exeter, EX6 8HD, U.K. T. +44(0) $1626891010-F+44(0) 1626891090$ - Email info@proteasome.com - 